\title{
Les bibliothèques publiques du Québec et le partage des ressources; ou l'éloge du saut en longueur
} Québec's Public Libraries and Resource Sharing, or in Praise of Long Jumping

\section{Las bibliotecas públicas de la provincia de Quebec y la repartición de los recursos; o el elogio del salto de longitud}

\section{Jacques Panneton}

Volume 39, numéro 4, octobre-décembre 1993

Les bibliothèques publiques

URI : https://id.erudit.org/iderudit/1033312ar

DOI : https://doi.org/10.7202/1033312ar

Aller au sommaire du numéro

Éditeur(s)

Association pour l'avancement des sciences et des techniques de la documentation (ASTED)

ISSN

0315-2340 (imprimé)

2291-8949 (numérique)

Découvrir la revue

Citer cet article

Panneton, J. (1993). Les bibliothèques publiques du Québec et le partage des ressources; ou l'éloge du saut en longueur. Documentation et bibliothèques, 39(4), 219-221. https://doi.org/10.7202/1033312ar
Résumé de l'article

Les contraintes financières et la mutation de l'environnement documentaire forcent les bibliothèques publiques du Québec au partage des ressources et à une réorientation stratégique. Les expériences québécoises en ce domaine sont très limitées, et l'exemple des bibliothèques publiques nord-américaines n'est pas concluant. L'avenir passe par un repositionnement créatif, fondé sur des expériences d'application partagée des nouvelles technologies, dans une perspective d'apport d'information additionnelle aux usagers, même si les bibliothèques paraissent ne pas en avoir les moyens. Investir, c'est choisir.
Tous droits réservés (C) Association pour l'avancement des sciences et des techniques de la documentation (ASTED),
Ce document est protégé par la loi sur le droit d'auteur. L'utilisation des services d'Érudit (y compris la reproduction) est assujettie à sa politique d'utilisation que vous pouvez consulter en ligne.

https://apropos.erudit.org/fr/usagers/politique-dutilisation/ 


\title{
Les bibliothèques publiques du Québec et le partage des ressources; ou l'éloge du saut en longueur
}

\author{
Jacques Panneton \\ Bibliothécaire en chef \\ Bibliothèque municipale de Montréal
}

Les contraintes financières et la mutation de l'environnement documentaire forcent les bibliothèques publiques du Québec au partage des ressources et à une réorientation stratégique. Les expériences québécoises en ce domaine sont très limitées, et l'exemple des bibliothèques publiques nord-américaines n'est pas concluant. L'avenir passe par un repositionnement créatif, fondé sur des expériences d'application partagée des nouvelles technologies, dans une perspective d'apport d'information additionnelle aux usagers, même si les bibliothèques paraissent ne pas en avoir les moyens. Investir, c'est choisir.

\section{Québec's Public Libraries and Resource Sharing, or in Praise of Long Jumping}

Financial cutbacks and changes in information formats and packages have forced public libraries in Québec to share their resources and to reorient their strategies. Such experiences in Québec are few in number and the North American public library experience is inconclusive. The future must rely on creative repositioning using the applications of new tecnologies in order to bring more information to users. Libraries may not have the resources to do it. To invest is to chose.
Las bibliotecas públicas de la provincia de Quebec y la repartición de los recursos; o el elogio del salto de longitud.

Las dificultades financieras y la mutación del medio ambiente documental fuerzan a las bibliotecas públicas de la provincia de Quebec a la repartición de los recursos y a una reorientación estratégica. Las experiencias quebequenses en este campo son muy limitadas, y el ejemplo de las bibliotecas públicas norteamericanas no es concluyente. El futuro deberá contar sobre la creatividad, fundada sobre las experiencias de aplicación compartida de nuevas tecnologías, con una perspectiva de aporte de la información adicional a los usuarios, aunque parezca que las bibliotecas no tengan los recursos financieros. Investir es escoger.
Plus que jamais les bibliothèques publiques québécoises sont interpellées sur le partage des ressources. Ceci tient pour une part à l'impact des déficits gouvernementaux qui se répercute de haut en bas, d'un niveau de gouvernement à l'autre, jusqu'à la base de la pyramide, les municipalités, qui ont l'obligation légale d'équilibrer leur budget. Les municipalités interrogent donc leurs bibliothèques publiques comme leurs autres services, surle niveau et l'efficience de leurs dépenses.

D'autre part, l'environnement documentaire traverse une période de mutation profonde qui voit l'apparition de nouveaux supports, avec la nécessité de les intégrer tout en faisant avec l'ancien; avec également des moyens financiers extrêmement limités.

Devant donc la forte pression résultant de l'augmentation des sources, des coûts, de la demande, et de la diminution des ressources financières, quelle peut être, quelle devrait êtrel'orientation stratégique des bibliothèques publiques québécoises?

En Amérique du Nord, et de plus en plus en Europe, le partage des ressources a graduellement acquis le statut de vertu théologale. Depuis une lettre de Samuel Green à l'éditeur de Library Journal en 1876, les initiatives américaines se sont multipliées et développées, des réseaux de coopération plus ou moins structurés se sont graduellement constitués jusqu'à l'apparition de réseaux mixtes incluant la participation de bibliothèques académiques, publiques, spécialisées, parfois même scolaires. À cet égard, l'article de Jean M. Oplinger constitue un témoignage fort intéressant ${ }^{1}$.

S'il existe un très large consensus sur la nécessité et l'avantage de partager expertise, infrastructures et ressources documentaires, on entend aussi quelques voix contester la rentabilité concrète des investissements consentis par les bibliothèques publiques américaines pour adhérer à ce mouvement. Par exemple, Thomas $\mathrm{H}$. Ballard effectue une analyse statistique troublante du prêt-entre-bibliothèques (PEB), certainement l'activité la plus courante de partage des ressources entre bibliothèques publiques ${ }^{2}$.

1. Jean M. Oplinger, «An Inquiry into Multitype Library Cooperation", Current Studies in Librarianship, (Spring/Fall 1991), 40-52.

2. Thomas H. Ballard, The Failure of Resource Sharing in Public Libraries and Alternative StrategiesforService, Chicago, AmericanLibrary Association, 1986. 


\section{L'échec du PEB}

En 1983, le prêt entre bibliothèques dans les bibliothèques publiques des ÉtatsUnis représentait 4804787 prêts sur 843 821572 , soit $0,57 \%^{3}$. Au Canada pour la même année, il représentait 956559 prêts sur 124243139 , soit $0,77 \%{ }^{4}$. Ballard souligne qu'à son avis, seul le prêt réciproque présente quelqu'intérêt. En effet, dans neuf systèmes américains et canadiens répertoriés, le prêt réciproque variait entre $1,63 \%$ et $8,1 \%$.

Pour ce qui est du Québec, nous disposons de peu d'études. Mais il est de notoriété publique que le partage des ressources s'y trouve à l'état embryonnaire, malgré l'existence de regroupements régionaux et certaines de leurs réalisations. L'étude de 1991 sur l'automatisation des bibliothèques publiques du Québec révèle que le $P E B$ québécois est de 12818 sur 28 millions de prêts $(0,04 \%)$. En Ontario, souvent citée en exemple dans le secteur des bibliothèques publiques, le $\mathrm{PEB}$ est plus élevé, à 350000 , mais fait tout de même seulement $0,5 \%$ du prêt total de 69 millions, soit à peu près la proportion américaine de $1983^{6}$.

Depuis une douzaine d'années, la Conférence des directeurs de bibliothèques publiques de l'île de Montréal opère un service de PEB. La moitié des bibliothèques membres de cette Conférence y participent. En 1991, il s'y est prêté 10813 documents sur un prêt total pour l'île de 8745768 , soit $0,12 \%$.

On pourrait discourir sur les causes de cette situation, discuter les entraves, contraintes et barrières diverses au PEB; se demander même s'il constitue un besoin réel pour l'usager des bibliothèques publiques. Àtout événement, l'expérience des bibliothèques publiques nord-américaines semble indiquer qu'il faut chercher ailleurs les voies de succès en matière de partage des ressources.

\section{Le jeu de position}

La transformation rapide de l'environnement documentaire pose un défi de taille à nos bibliothèques publiques: leur repositionnement dans le réseau d'information. Il peut être l'occasion d'un bond en avant si elles procèdent aux bons investissements. La Bibliothèque Nationale du Canada souligne avec justesse que le processus de mise en commun des ressources est parfois déterminé davantage par les développements technologiques et leurs applications pratiques que par des considérations philosophiques. En effet, par suite de l'apparition du CD-ROM ... et de l'avènement de l'édition électronique, la nature des ressources. mêmes est en train de changer?.

La disparition des fonctions traditionnelles des bibliothèques publiques n'est pas pour demain, particulièrement dans l'axe de la culture et du loisir. En matière d'éducation et d'information cependant, il en va autrement et l'émergence de la bibliothèque virtuelle (ou immatérielle, selon l'expression française) va grandement affecter la perception de la bibliothèque par les usagers d'information.

Après le CD-ROM, ce sont les terminaux multimédias (écrit, image, son), l'édition électronique, le courrier électronique, l'accès électronique au plein texte, la numérisation des documents, les postes de travail intégrant l'importation de données, la transformation de données et le traitement de texte. La prochaine génération d'usagers de nos bibliothèques sera rompue à l'utilisation de ces moyens. Déjà, les enseignants voient leurs élèves leur remettre des devoirs réalisés avec Windows sur l'ordinateur familial.

Les bibliothèques publiques $d u$ Québec doivent effectuer une reconversion substantielle et intégrer ces possibilités, sous peine que leur fonction d'information disparaisse de la perception collective, sous peine donc de disparaître du marché de l'information. L'automatisation de l'information bibliographique, quoique nécessaire, ne suffira pas. II faut y ajouter la gestion et la diffusion électroniques de l'information.

La transformation doit être assurée dans le respect des valeurs propres aux bibliothèques publiques, bien qu'elle implique de grands défis, notamment au plan de la formation des usagers:

Another scenario is the public library that develops a plan to transition into the electronic networked environment from their current traditional environment. While embracing and exploiting networkedinformation services and resources, it also maintains the high visibility and high demand traditional services ${ }^{8}$.

The evolving role for the public library in the networked environment can incorporate the traditional safety net role that ensures access to the network by all citizens. But its role should also expand into "electronic navigatorand intermediary», it should be "provider of electronic information to remote users", "coordinator of local community electronic information ressources», and "switching station for electronic information resources and services ${ }^{9}$.

Plusieurs conditions de succès sont déjà réunies. L'automatisation des bibliothèques publiques est bien amorcée $(38 \%)$ et se continue. Les serveurs de notices bibliographiques existent. Une modélisation du réseau automatisé de bibliothèques publiques québécoises est effectuée. Leministère de la Culture privilégie la coopération et le partage des ressources dans le cadre de sa politique culturelle $e^{10}$. Les bibliothèques publiques québécoises sont réunies dans deux regroupements nationaux et onze regroupements régionaux. Une expertise significative est déjà accumulée dans nos bibliothèques publiques.

3. Ibid., p. 39.

4. Ibid., p. 39.

5. Ibid., p. 52 .

6. Étude sur l'informatisation des bibliothèques publiques du Québec présentée à l'Association des directeurs de bibliothèques publiques $d u$ Québec (ADIBIPUQ), Montréal, Conseillers en gestion et informatique CGI Inc., 1991, p. 35.

7. La mise en commun des ressources au Canada $=$ Resources Sharing in Canada, Ottawa, Bibliothèque nationale du Canada, 1990, p. 62.

8. Charles R. McClure et al., Public Libraries and the INTERNET/NREN, Syracuse, N.Y., School of Information Studies, Syracuse University, 1992, p.28.

9. Ibid., p. 29.

10. Québec, Ministère des Affaires culturelles, La Politique culturelle du Québec, Québec, le Ministère, Direction des communications, $1992,150 \mathrm{p}$. 


\section{L'obligation de sauter}

La pire stratégie serait d'attendre, de se fixer sur les contraintes (ignorées ici à dessein) et de laisser la crise financière des gouvernements paralyser l'imagination et l'initiative. Certaines alternatives sont possibles.

D'abord, les bibliothèques publiques québécoises doivent compter sur leurs propres moyens. Ceci doit être une attitude. II n'y a plus d'argent, les gouvernements se chamaillent sur la décentralisation et sur leurs juridictions, rêvent de se désengager le plus possible.

Ensuite, les bibliothèques publiques doivent prendre le ministère de la Culture au mot et le presser de donner suite à court terme aux recommandations de l'Étude sur l'informatisation des bibliothèques publiques du Québec. Au besoin, que les bibliothèques lui soumettent elles-mêmes un projet pilote. Ceci permettrait une mise en commun rapide des services catalographiques et bibliographiques, la discipline par les pairs de l'autarcie en matière de traitement documentaire, et l'émergence d'un laboratoire de rapprochement entre les bibliothèques autonomes et les centres régionaux de services aux bibliothèques publiques (les ex-BCP).
Et encore, les bibliothèques doivent se payer des petites expériences d'application partagée des nouvelles technologies; par exemple, l'exploitation en résẻau de serveurs CD-ROM, ou de banques d'informations numérisées. Puisque chaque bibliothèque est confrontée au défi du repositionnement, aussi bien le faire ensemble, à condition d'investir dans des services apportant aux usagers un afflux additionnel d'informations utiles. Qu'elles oublient un peu le PEB et le transfert physique de documents, et songent un peu plus au transfert électronique de l'information et au prêt réciproque, c'est-àdire à la possibilité pour l'usager d'emprunter le document directement de la bibliothèque qui le détient, sans intermédiaires coûteux: s'il existe une marge financière, qu'elles l'utilisent au bon endroit. Au besoin, que nos bibliothèques se préparent à des réallocations internes de ressources. Bien qu'il soit de bonne guerre de donner au ministère de la Culture une occasion de supporter financièrement de telles initiatives, il faut refuser d'en dépendre.

Enfin, les bibliothèques publiques doivent jouer le jeu des ententes régionales de développement culturel prévues dans la politique culturelle du Québec. Dans les domaines de la promotion de la lecture et de l'animation, ceci devrait se faire avec des partenaires externes au monde documentaire, permettant ainsi l'émergence de solidarités nouvelles dans un contexte de coopération élargie.

\section{Le rétablissement}

Où cela nous mène-t-il? Bien malin qui est sûr de la réponse quand il nage en pleine révolution. Le besoin crée l'organe, et nécessité fait loi. Deux banalités pour exprimer qu'il vaut mieux aller ailleurs que rester ici, quand ici peut devenir nulle part.

Les bibliothèques publiques sont un acquis très important des sociétés libérales. Encore aujourd'hui elles expriment la conviction du rôle irremplaçable de l'écrit et de l'information dans le développement démocratique des collectivités. Elles demeureront une présence active dans la mesure où elles s'adapteront aux changements de l'environnement, non pas en renonçant aux valeurs qu'elles représentent, mais en redéployant leurs moyens en bonne intelligence de cet environnement.

Le partage des ressources implique le partage des valeurs. Une approche expérimentale de partage fondée sur une vision stratégique commune permettra au déploiement concerté des ressources de tomber avec naturel sur une piste transformée. Lechangement requis nécessite peutêtre du renoncement mais pas une renonciation.

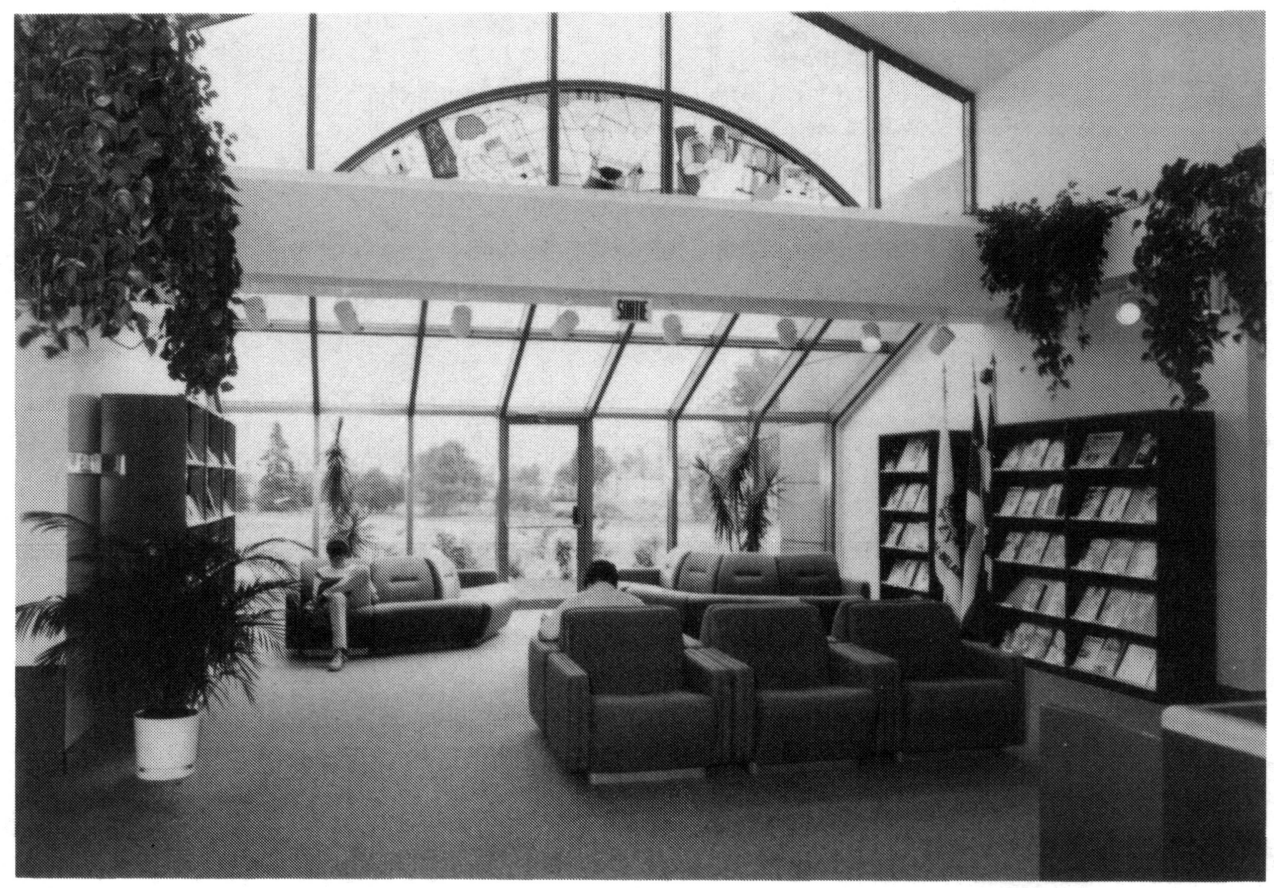

Coup d'oeil sur la bibliothèque Félix-Leclerc à Val-Bélair 